\title{
Recent BES Results on Hadron Spectroscopy
}

\author{
Chang-Zheng Yuan (for the BES Collaboration) ${ }^{\mathrm{a} *}$ \\ ${ }^{a}$ Institute of High Energy Physics, Chinese Academy of Sciences, \\ P.O. Box 918-1, Beijing 100049, China
}

We present recent results from the BES experiment on the observation of the $Y(2175)$ in $J / \psi \rightarrow \phi f_{0}(980) \eta$, study of $\eta(2225)$ in $J / \psi \rightarrow \gamma \phi \phi$, and the production of $X(1440)$ recoiling against an $\omega$ or a $\phi$ in $J / \psi$ hadronic decays. The observation of $\psi(2 S)$ radiative decays is also presented.

\section{Introduction}

The analyses reported in this talk were performed using either a sample of $58 \times 10^{6} \mathrm{~J} / \psi$ events or a sample of $14 \times 10^{6} \psi(2 S)$ events collected with the upgraded Beijing Spectrometer (BESII) detector [1] at the Beijing ElectronPositron Collider (BEPC).

\section{The $Y(2175)$ in $J / \psi \rightarrow \phi f_{0}(980) \eta[2$}

A new structure, denoted as $Y(2175)$ and with mass $m=2.175 \pm 0.010 \pm 0.015 \mathrm{GeV} / c^{2}$ and width $\Gamma=58 \pm 16 \pm 20 \mathrm{MeV} / c^{2}$, was observed by the BaBar experiment in the $e^{+} e^{-} \rightarrow \gamma_{I S R} \phi f_{0}(980)$ initial-state radiation process [34]. This observation stimulated some theoretical speculation that this $J^{P C}=1^{--}$state may be an $s$-quark version of the $Y(4260)$ since both of them are produced in $e^{+} e^{-}$annihilation and exhibit similar decay patterns [5].

Here we report the observation of the $Y(2175)$ in the decays of $J / \psi \rightarrow \eta \phi f_{0}(980)$, with $\eta \rightarrow \gamma \gamma$, $\phi \rightarrow K^{+} K^{-}, f_{0}(980) \rightarrow \pi^{+} \pi^{-}$. A four-constraint energy-momentum conservation kinematic fit is performed to the $K^{+} K^{-} \pi^{+} \pi^{-} \gamma \gamma$ hypothesis for the selected four charged tracks and two photons. $\eta \rightarrow \gamma \gamma$ candidates are defined as $\gamma$-pairs with $\left|M_{\gamma \gamma}-0.547\right|<0.037 \mathrm{GeV} / c^{2}$, a $\phi$ signal

\footnotetext{
${ }^{*}$ Invited talk at the International workshop on $e^{+} e^{-}$collisions from Phi to Psi, Laboratori Nazionali di Frascati, Italy, 7-10 April, 2008. Supported in part by the 100 Talents Program of CAS under Contract No. U-25 and by National Natural Science Foundation of China under Contract No. 10491303.
}

is defined as $\left|m_{K^{+} K^{-}}-1.02\right|<0.019 \mathrm{GeV} / c^{2}$, and in the $\pi^{+} \pi^{-}$invariant mass spectrum, candidate $f_{0}(980)$ mesons are defined by $\mid m_{\pi^{+} \pi^{-}}-$ $0.980 \mid<0.060 \mathrm{GeV} / c^{2}$. The $\phi f_{0}(980)$ invariant mass spectrum for the selected events is shown in Fig. 1] where a clear enhancement is seen around $2.18 \mathrm{GeV} / c^{2}$. Fit with a Breit-Wigner and a polynomial background yields $52 \pm 12$ signal events and the statistical significance is found to be $5.5 \sigma$ for the signal. The mass of the structure is determined to be $M=2.186 \pm$ 0.010 (stat) \pm 0.006 (syst) $\mathrm{GeV} / c^{2}$, the width is $\Gamma=0.065 \pm 0.023$ (stat) \pm 0.017 (syst) $\mathrm{GeV} / c^{2}$, and the product branching ratio is $\mathcal{B}(J / \psi \rightarrow$ $\eta Y(2175)) \cdot \mathcal{B}\left(Y(2175) \rightarrow \phi f_{0}(980)\right) \cdot \mathcal{B}\left(f_{0}(980) \rightarrow\right.$ $\left.\pi^{+} \pi^{-}\right)=(3.23 \pm 0.75($ stat $) \pm 0.73($ syst $)) \times 10^{-4}$. The mass and width are consistent with BaBar's results.

\section{The $\eta(2225)$ in $J / \psi \rightarrow \gamma \phi \phi[7]$}

Structures in the $\phi \phi$ invariant-mass spectrum have been observed by several experiments both in the reaction $\pi^{-} p \rightarrow \phi \phi n$ [8] and in radiative $J / \psi$ decays 91011. The $\eta(2225)$ was first observed by the MARK-III collaboration in $J / \psi$ radiative decays $J / \psi \rightarrow \gamma \phi \phi$. A fit to the $\phi \phi$ invariant-mass spectrum gave a mass of $2.22 \mathrm{GeV} / c^{2}$ and a width of $150 \mathrm{MeV} / c^{2}$ 9. An angular analysis of the structure found it to be consistent with a $0^{-+}$assignment. It was subsequently observed by the DM2 collaboration, also in $J / \psi \rightarrow \gamma \phi \phi$ decays [1011.

We present results from a high statistics study 


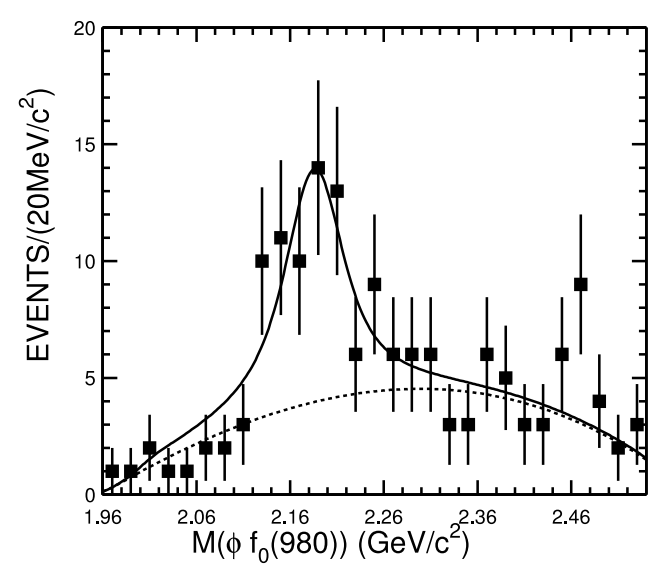

Figure 1. The $\phi f_{0}(980)$ invariant mass distribution of the data (points with error bars) and the fit (solid curve) with a Breit-Wigner function and polynomial background; the dashed curve indicates the background function.

of $J / \psi \rightarrow \gamma \phi \phi$ in the $\gamma K^{+} K^{-} K_{S}^{0} K_{L}^{0}$ final state, with the $K_{L}^{0}$ missing and reconstructed with a one-constraint kinematic fit. After kinematic fit, we require both the $K^{+} K^{-}$and $K_{S}^{0} K_{L}^{0}$ invariant masses lie within the $\phi$ mass region $\left(\left|M\left(K^{+} K^{-}\right)-m_{\phi}\right|<12.5 \mathrm{MeV} / c^{2}\right.$ and $\left.\left|M\left(K_{S}^{0} K_{L}^{0}\right)-m_{\phi}\right|<25 \mathrm{MeV} / c^{2}\right)$. The $\phi \phi$ invariant mass distribution is shown in Fig. 2, There are a total of 508 events with a prominent structure around $2.24 \mathrm{GeV} / c^{2}$.

A partial wave analysis of the events with $M(\phi \phi)<2.7 \mathrm{GeV} / c^{2}$ was performed. The twobody decay amplitudes in the sequential decay process $J / \psi \rightarrow \gamma X, X \rightarrow \phi \phi, \phi \rightarrow K^{+} K^{-}$and $\phi \rightarrow K_{S}^{0} K_{L}^{0}$ are constructed using the covariant helicity coupling amplitude method. The intermediate resonance $X$ is described with the normal Breit-Wigner propagator $B W=1 /\left(M^{2}-\right.$ $s-i M \Gamma)$, where $s$ is the $\phi \phi$ invariant masssquared and $M$ and $\Gamma$ are the resonance's mass and width. When $J / \psi \rightarrow \gamma X, X \rightarrow \phi \phi$ is fitted with both the $\phi \phi$ and $\gamma X$ systems in a $P$ wave, which corresponds to a pseudoscalar $X$ state, the fit gives $196 \pm 19$ events with mass $M=2.24_{-0.02-0.02}^{+0.03+0.03} \mathrm{GeV} / c^{2}$, width $\Gamma=0.19 \pm$

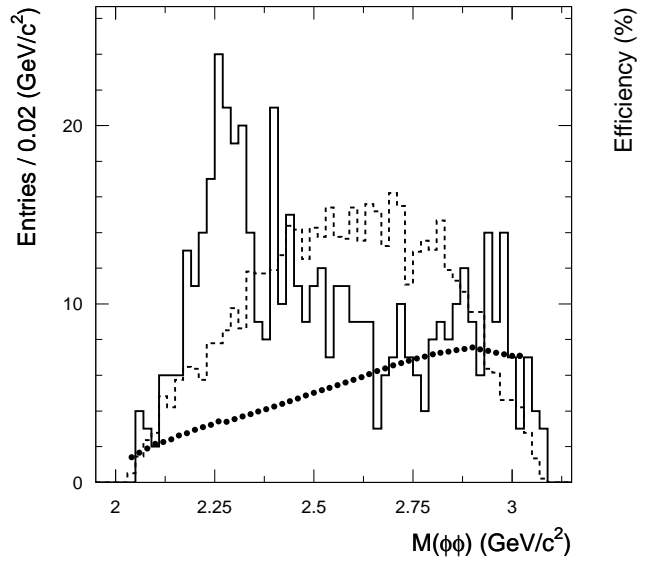

Figure 2. The $K^{+} K^{-} K_{S}^{0} K_{L}^{0}$ invariant mass distribution for $J / \psi \rightarrow \gamma \phi \phi$ candidate events. The dashed histogram is the phase space invariant mass distribution, and the dotted curve indicates how the acceptance varies with the $\phi \phi$ invariant mass.

$0.03_{-0.06}^{+0.04} \mathrm{GeV} / c^{2}$, and a statistical significance larger than $10 \sigma$, and a product branching fraction of: $\mathcal{B}(J / \psi \rightarrow \gamma \eta(2225)) \cdot \mathcal{B}(\eta(2225) \rightarrow \phi \phi)=$ $(4.4 \pm 0.4 \pm 0.8) \times 10^{-4}$.

The presence of a signal around $2.24 \mathrm{GeV} / c^{2}$ and its pseudoscalar character are confirmed, and the mass, width, and branching fraction are in good agreement with previous experiments.

\section{The $X(1440)$ in $J / \psi$ hadronic decays 12}

A pseudoscalar gluonium candidate, the socalled $E / \iota(1440)$, was observed in $p \bar{p}$ annihilation in 1967 [13 and in $J / \psi$ radiative decays in the 1980's 141516. The study of the decays $J / \psi \rightarrow\{\omega, \phi\} K \bar{K} \pi$ is a useful tool in the investigation of quark and possible gluonium content of the states around $1.44 \mathrm{GeV} / c^{2}$. Here we investigate the possible structure in the $K \bar{K} \pi$ final state in $J / \psi$ hadronic decays at around $1.44 \mathrm{GeV} / c^{2}$.

In this analysis, $\omega$ mesons are observed in the $\omega \rightarrow \pi^{+} \pi^{-} \pi^{0}$ decay, $\phi$ mesons in the $\phi \rightarrow K^{+} K^{-}$ decay, and other mesons are detected in the decays: $K_{S}^{0} \rightarrow \pi^{+} \pi^{-}, \pi^{0} \rightarrow \gamma \gamma$. KK $\bar{K} \pi$ could be 
$K_{S}^{0} K^{ \pm} \pi^{\mp}$ or $K^{+} K^{-} \pi^{0}$.

Figures 3 and 4 show the $K_{S}^{0} K^{ \pm} \pi^{\mp}$ and $K^{+} K^{-} \pi^{0}$ invariant mass spectra after $\omega$ selection $\left(\left|m_{\pi^{+} \pi^{-} \gamma \gamma}-m_{\omega}\right|<0.04 \mathrm{GeV} / \mathrm{c}^{2}\right)$ or $\phi$ signal selection $\left(\left|m_{K^{+} K^{-}}-m_{\phi}\right|<0.015 \mathrm{GeV} / c^{2}\right)$. Clear $X(1440)$ signal is observed recoiling against the $\omega$, and there is no significant signal recoiling against a $\phi$.

The $K_{S}^{0} K^{ \pm} \pi^{\mp}$ invariant mass distribution in $J / \psi \rightarrow \omega K_{S}^{0} K^{ \pm} \pi^{\mp}$ (Fig. 3(b)) is fitted with a BW function convoluted with a Gaussian mass resolution function $\left(\sigma=7.44 \mathrm{MeV} / c^{2}\right)$ to represent the $X(1440)$ signal and a third-order polynomial background function. The mass and width obtained from the fit are $M=1437.6 \pm$ $3.2 \mathrm{MeV} / c^{2}$ and $\Gamma=48.9 \pm 9.0 \mathrm{MeV} / c^{2}$, and the fit yields $249 \pm 35$ events. Using the efficiency of $1.45 \%$ determined from a uniform phase space MC simulation, we obtain the branching fraction to be $\mathcal{B}(J / \psi \rightarrow \omega X(1440)) \cdot \mathcal{B}(X(1440) \rightarrow$ $K_{S}^{0} K^{+} \pi^{-}+$c.c. $)=(4.86 \pm 0.69 \pm 0.81) \times 10^{-4}$, where the first error is statistical and the second one systematic.

For $J / \psi \rightarrow \omega K^{+} K^{-} \pi^{0}$ mode, by fitting the $K^{+} K^{-} \pi^{0}$ mass spectrum in Fig. 3(c) with same functions, we obtain the mass and width of $M=$ $1445.9 \pm 5.7 \mathrm{MeV} / c^{2}$ and $\Gamma=34.2 \pm 18.5 \mathrm{MeV} / c^{2}$, and the number of events from the fit is $62 \pm 18$. The efficiency is determined to be $0.64 \%$ from a phase space $\mathrm{MC}$ simulation, and the branching fraction is $\mathcal{B}(J / \psi \rightarrow \omega X(1440)) \cdot \mathcal{B}(X(1440) \rightarrow$ $\left.K^{+} K^{-} \pi^{0}\right)=(1.92 \pm 0.57 \pm 0.38) \times 10^{-4}$, in good agreement with the isospin symmetry expectation from $J / \psi \rightarrow \omega K_{S}^{0} K^{ \pm} \pi^{\mp}$ mode.

The distribution of $K_{S}^{0} K^{ \pm} \pi^{\mp}$ and $K^{+} K^{-} \pi^{0}$ invariant mass spectra recoiling against the $\phi$ signal are shown in Fig. 4, and there is no evidence for $X(1440)$. The upper limits on the branching fractions at the $90 \%$ C.L. are $\mathcal{B}(J / \psi \rightarrow \phi X(1440) \rightarrow$ $\phi K_{S}^{0} K^{+} \pi^{-}+$c.c. $)<1.93 \times 10^{-5}$ and $\mathcal{B}(J / \psi \rightarrow$ $\left.\phi X(1440) \rightarrow \phi K^{+} K^{-} \pi^{0}\right)<1.71 \times 10^{-5}$.

In conclusion, the mass and width of the $X(1440)$ are measured, which are in agreement with previous measurements; the branching fractions we measured are also in agreement with the DM2 and MARK-III results. The significant signal in $J / \psi \rightarrow \omega K \bar{K} \pi$ mode and the missing signal in $J / \psi \rightarrow \phi X$ mode may indicate the $s \bar{s}$ compo- nent in the $X(1440)$ is not significant.

\section{5. $\psi(2 S)$ radiative decays}

Besides conventional meson and baryon states, QCD also predicts a rich spectrum of glueballs, hybrids, and multi-quark states in the 1.0 to $2.5 \mathrm{GeV} / c^{2}$ mass region. Therefore, searches for the evidence of these exotic states play an important role in testing QCD. The radiative decays of $\psi(2 S)$ to hadrons are expected to contribute about $1 \%$ to the total $\psi(2 S)$ decay width 17 . However, the measured channels only sum up to about $0.05 \%$ [18.

We measured the decays of $\psi(2 S)$ into $\gamma p \bar{p}$, $\gamma 2\left(\pi^{+} \pi^{-}\right), \quad \gamma K_{S}^{0} K^{+} \pi^{-}+$c.c.,$\gamma K^{+} K^{-} \pi^{+} \pi^{-}$, $\gamma K^{* 0} K^{-} \pi^{+}+$c.c. $, \quad \gamma K^{* 0} \bar{K}^{* 0}, \quad \gamma \pi^{+} \pi^{-} p \bar{p}$, $\gamma 2\left(K^{+} K^{-}\right), \gamma 3\left(\pi^{+} \pi^{-}\right)$, and $\gamma 2\left(\pi^{+} \pi^{-}\right) K^{+} K^{-}$, with the invariant mass of the hadrons $\left(m_{h s}\right)$ less than $2.9 \mathrm{GeV} / c^{2}$ for each decay mode 19 . The differential branching fractions are shown in Fig. 5. The branching fractions below $m_{h s}<2.9 \mathrm{GeV} / c^{2}$ are given in Table 1] which sum up to $0.26 \%$ of the total $\psi(2 S)$ decay width. We also analyzed $\psi(2 S) \rightarrow \gamma \pi^{+} \pi^{-}$and $\gamma K^{+} K^{-}$ modes to study the resonances in $\pi^{+} \pi^{-}$and $\mathrm{K}^{+} \mathrm{K}^{-}$invariant mass spectrum. Significant signals for $f_{2}(1270)$ and $f_{0}(1710)$ were observed, but the low statistics prevent us from drawing solid conclusion on the other resonances [20].

\section{Summary}

Using the $58 \mathrm{M} J / \psi$ and $14 \mathrm{M} \psi(2 S)$ events samples taken with the BESII detector at the $\mathrm{BEPC}$ storage ring, BES experiment provided many interesting results in charmonium decays, including the observation of the $Y(2175)$, $\eta(2225), X(1440)$, and many $\psi(2 S)$ radiative decays. These results shed light on the understanding of strong interaction sector of the Standard Model.

\section{REFERENCES}

1. J. Z. Bai et al. [BES Collaboration], Nucl. Instr. Meth. A 458, 627 (2001).

2. M. Ablikim et al. [BES Collaboration], Phys. Rev. Lett. 100, 102003 (2008). 

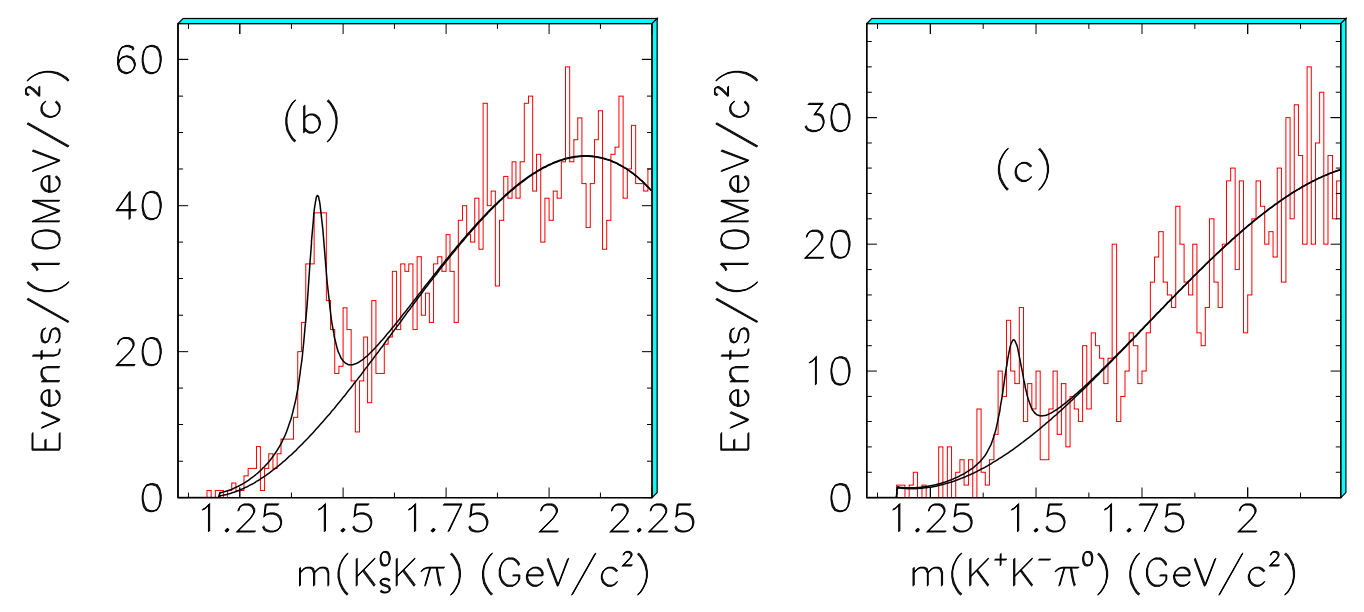

Figure 3. The $K \bar{K} \pi$ invariant mass distribution for $J / \psi \rightarrow \omega K_{S}^{0} K^{ \pm} \pi^{\mp}$ (b) and $\omega K^{+} K^{-} \pi^{0}$ (c) candidate events. The curves are the best fit.
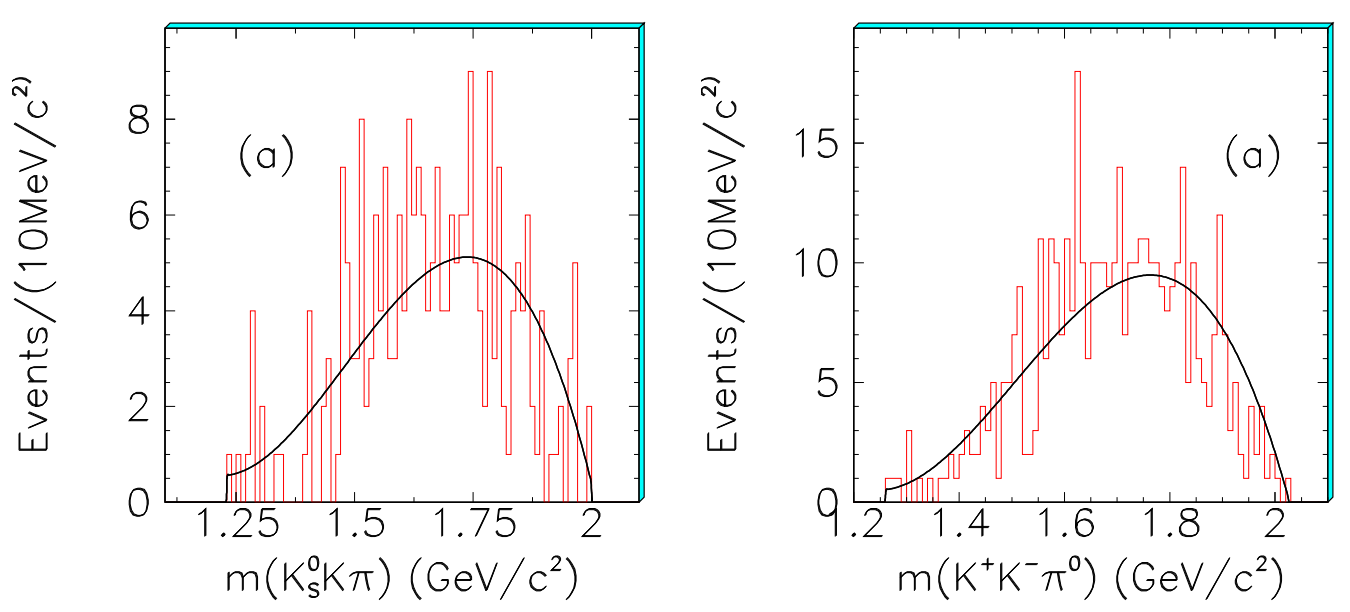

Figure 4. The $K_{S}^{0} K^{ \pm} \pi^{\mp}$ (left) and $K^{+} K^{-} \pi^{0}$ (right) invariant mass recoiling against the $\phi$ in $J / \psi \rightarrow$ $\phi K \bar{K} \pi$ mode. 


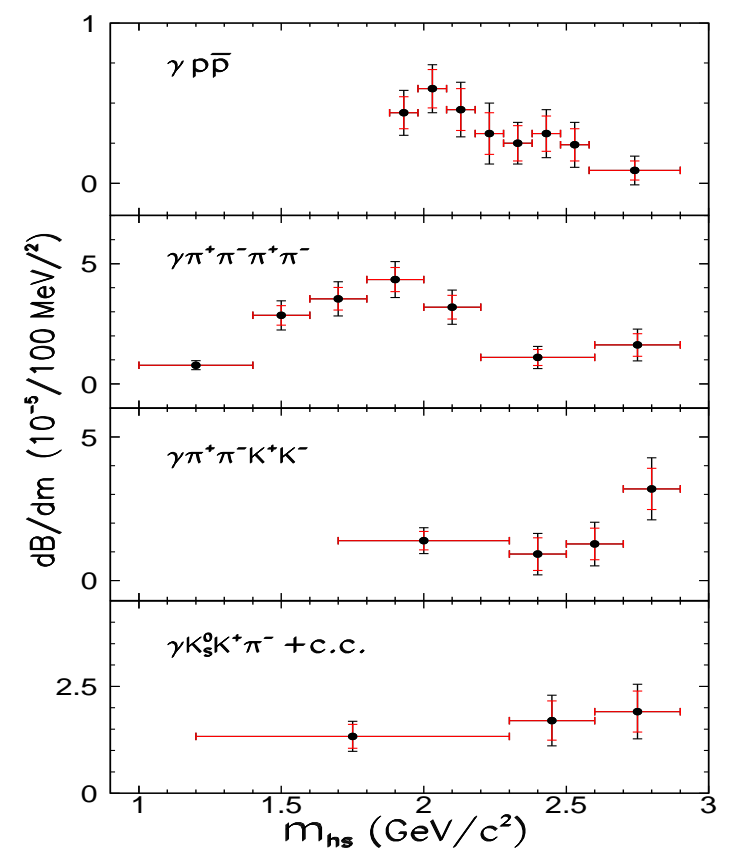

Figure 5. Differential branching fractions for $\psi(2 S) \rightarrow \gamma p \bar{p}, \gamma 2\left(\pi^{+} \pi^{-}\right), \gamma K^{+} K^{-} \pi^{+} \pi^{-}$, and $\gamma K_{S}^{0} K^{+} \pi^{-}+$c.c. . Here $m_{h s}$ is the invariant mass of the hadrons. For each point, the smaller longitudinal error is the statistical error, while the bigger one is the total error.

\section{Table 1}

Branching fractions for $\psi(2 S) \rightarrow \gamma+$ hadrons with $m_{h s}<2.9 \mathrm{GeV} / c^{2}$, where the upper limits are determined at the $90 \%$ C.L.

\begin{tabular}{ll}
\hline \hline Mode & $\mathcal{B}\left(\times 10^{-5}\right)$ \\
\hline$\gamma p \bar{p}$ & $2.9 \pm 0.4 \pm 0.4$ \\
$\gamma 2\left(\pi^{+} \pi^{-}\right)$ & $39.6 \pm 2.8 \pm 5.0$ \\
$\gamma K_{S}^{0} K^{+} \pi^{-}+c . c$. & $25.6 \pm 3.6 \pm 3.6$ \\
$\gamma K^{+} K^{-} \pi^{+} \pi^{-}$ & $19.1 \pm 2.7 \pm 4.3$ \\
$\gamma K^{* 0} K^{+} \pi^{-}+$c.c. & $37.0 \pm 6.1 \pm 7.2$ \\
$\gamma K^{* 0} \bar{K}^{* 0}$ & $24.0 \pm 4.5 \pm 5.0$ \\
$\gamma \pi^{+} \pi^{-} p \bar{p}$ & $2.8 \pm 1.2 \pm 0.7$ \\
$\gamma K^{+} K^{-} K^{+} K^{-}$ & $<4$ \\
$\gamma 3\left(\pi^{+} \pi^{-}\right)$ & $<17$ \\
$\gamma 2\left(\pi^{+} \pi^{-}\right) K^{+} K^{-}$ & $<22$ \\
\hline \hline
\end{tabular}

3. B. Aubert et al. [BABAR Collaboration], Phys. Rev. D 74, 091103 (2006).

4. B. Aubert et al. [BABAR Collaboration], Phys. Rev. D 76, 012008 (2007).

5. B. Aubert et al. [BaBar Collaboration], Phys. Rev. Lett. 95, 142001 (2005).

6. C. Z. Yuan et al. [Belle Collaboration], Phys. Rev. Lett. 99, 182004 (2007).

7. M. Ablikim et al. [BES Collaboration], Phys. Lett. B 662, 330 (2008).

8. A. Etkin et al., Phys. Lett. B 201, 568 (1988).

9. Z. Bai et al. [MARK-III Collaboration], Phys. Rev. Lett. 65, 1309 (1990).

10. D. Bisello et al. [DM2 Collaboration], Phys. Lett. B 179, 294 (1986).

11. D. Bisello et al. [DM2 Collaboration], Phys. Lett. B 241, 617 (1990).

12. M. Ablikim et al. [BES Collaboration], Phys. Rev. D 77, 032005 (2008).

13. P. H. Baillon et al., NC 50A, 393 (1967).

14. D. L. Scharre et al., Phys. Lett. B 97, 329 (1980).

15. C. Edwards et al., Phys. Rev. Lett. 49, 259 (1982); Phys. Rev. Lett. 50, 219 (1982).

16. J. E. Augustin et al. [DM2 Collaboration], Phys. Rev. D 42, 10 (1990).

17. P. Wang, C. Z. Yuan, and X. H. Mo, Phys. Rev. D 70, 114014 (2004).

18. W.-M. Yao et al. [Particle Data Group], J. Phys. G 33, 1 (2006).

19. M. Ablikim et al. [BES Collaboratio], Phys. Rev. Lett. 99, 011802 (2007).

20. M. Ablikim et al. [BES Collaboration], arXiv:0710.2324 [hep-ex]. 